\title{
Die Einwirkung von homogenisiertem Lungengewebe auf Tween 20
}

\author{
Von \\ W. Lührs und K. Chrometzka \\ Alıs dem Sanatorium "Bergfrieden" - Rottach-Egern der Arbeitsgemeinschaft fïr Krebsbekämpfung, Sitz. Bocbum -
Rubrknappschaft (Chefarzt: Prof. Dr. W. Lübrs)
}

(Der Schriftleitung zugegangen am 28. November 1963)

\begin{abstract}
Manometrische Messungen lassen den Schluß zu, daß die Lipase homogenisierten Lungengewebes „Tween 20 “ vcrseift und Fettsäure (Laurinsäure) freisetzt.

Manometric measurements show that the lipase of homogenised lung tissue hydrolyses „Tween 20 “ and releases a fatty acid (lauric acid).
\end{abstract}

Nach dem letzten Weltkrieg wurde eine große Gruppe von oberflächenaktiven, nicht polaren Substanzen, Emulgatoren, Detergentien und anderen Substanzen für die verschiedensten $Z$ wecke nutzbar gemacht. Diese Substanzen fanden Anwendung als Zusatzstoffe bei Nahrungsmitteln, in der Kosmetik, bei Pflanzenschutzmitteln, in der Textilindustrie, in der chemischen Reinigung, in der Mineralölindustrie, bei der Zusammenstellung von Polituren und Putzmitteln und auch in der Pharmazie (1).

Nachdem seit dem Jahre 1941 bis in die jüngste Zeit hinein Berenblum (2) nachweisen konnte, daß der $\mathrm{Zu}$ satz von Crotonöl zu bestimmten hautaktiven karzinogenen Substanzen eine deutliche Steigerung des Angehens von Hauttumoren erkennen läßt, wurden andere Substanzen ähnlicher Eigenschaft als Adjuvans geprüft. SETÄLÄ (3-5) fand seit 1956 immer wieder, daß die Polyoxyäthylen-sorbitan-monolaurate einen ähnlichen Effekt innerhalb der Kokanzerogenese bewirken wie das Crotonöl. Im Gegensatz dazu fanden Berenblum und HARAN (6), daß Gaben von 9, 10-Dimethyl-1,2benzanthrazen, 3,4-Benzpyren, 20-Methylcholanthren oder 1, 2, 5, 6,-Dibenzanthrazen mit Schlundsonde bei männlichen Siviss- und $\mathrm{C}_{3} \mathrm{H}$-Mäusen zu Tumoren des Vormagens führten. Die zusätzliche Einwirkung von Crotonöl führte zu einer Zunahme des Angehens der Tumoren, wobei die Autoren eine Summation der karzinogenen Wirkung des Kohlenwasserstoffes und des Crotonöles annehmen. Entsprechende Versuche mit einem Polyäthylenglykol ließen keinen krebsfördenden Effekt erkennen.

Andererseits fanden Wong, JURAS und WissLer (7), daß oral verabreichtes Tween 80 und Methylcholanthren bei Mäusen eine Zunahme der Tumorzahl im Vormagen, zum anderen eine Zunahme der Tumorzahl in verschiedenen Organen ergab und eine deutliche Zunahme multipler Tumoren nachweisen ließ. Es wurde eine partielle Tween-Resorption angedeutet. Dieses Ergebnis zeigte einen echten Unterschied gegenüber den Tierversuchen mit einer alleinigen oralen Gabe von Methylcholanthren.

Im Gegensatz zu diesen Untersuchungen berichteten Shubik, Della Porta und Spencer (8) über Versuche mit Tween 60 als kokanzerogener Substanz. Sie fanden, daß diese oberflächenaktive Substanz wahrscheinlich kokanzerogene Eigenschaften besitzen kann, aber unwesentlich für eine kausale Kanzerogenese ist. HuEper und Mitarbeiter (9) kamen auf Grund ihrer ticrexperimentellen Untersuchungen zu dem Ergebnis, daß die Polyoxyäthylenderivate besonders als Stearate keinen sicheren Einfluß auf das Entstehen von primären Blasenkarzinomen crkennen ließen. Mit großer Wahrscheinlichkeit können diese oberflächenaktiven Substanzen nicht als primäre Karzinogene bezeichnet werden. Interessant war in diesem Zusammenhang, daß manche Pharmaka, die gerade bei chronischer Bronchitis und ähnlichen Indikationen von der pharmazeutischen Industrie angeboten werden, diese oberflächenaktiven Stoffe - besonders Tween 20 - enthalten.

Interessant war das Verhalten dieses Tween $20 \mathrm{im} \mathrm{Zu-}$ sammenwirken mit Lungengewebe. Die Hypothese einer möglichen enzymatischen Einwirkung von Lungengewebe auf Fettsubstanzen geht bereits auf das Jahr 1908-(Sieber, 10) zurück. Bradley (11) und Hamsik (12) fanden neben einer relativ starken lipolytischen Eigenschaft des Lungengewebes auch eine synthetisierende Wirkung. Vom biochemischen Standpunkt hat die Frage nach der Lungenlipase großes Interesse gefunden, besonders im Zusammenhang mit der Tuberkulose. In der Lunge finden sich neben der Lipase noch drei grundsätzlich verschiedene Esterasen. Es ist die Esterase im engeren Sinn, die Cholinesterase und die Acetylcholinesterase. Die Lipase spaltet vor allem langkettige Fettsäureester, die Esterase kurzkettige Fettsäureester (13). Wir haben den Einfluß von frisch gewonnenem Lungengewebe von Kaninchen in der Warburg-Apparatur auf Tween $2^{1}$ ) in verschiedenen Konzentrationen untersucht (14).

\section{Methodik}

Wir nahmen an, da $\beta$ in frischer Kaninchenlunge enthaltene Lipase Tween 20 verseifen kann. Die freigesetzte Laurinsäure treibt aus einer gepufferten bikarbonathaltigen Ringerlösung Kohlensäure aus, die manometrisch gemessen wird.

1) Wir danken der Firma Dr. Schwarz, Monheim, für die Überlassung der Versuchsmengen "Tween 20" (Polyoxyäthylen-sorbitan-monolaurat). 
Frische Kaninchenlunge wurde zu Brei zerkleinert und anschließend im Potter-Elvehjem homogenisiert. $5 \mathrm{~g}$ des Homogenates wurden in dest. Wasser im Verhältnis 1:3 aufgenommen. Pro Ansatz wurden $0,5 \mathrm{~m} l$ des wäßr. Homogenates eingesetzt. Die Messungen wurden mit einer Tween-Konzentration von $0,407-0,0008 \mathrm{mMol}$ vorgenommen. Das im Versuch zur Anwendung gekommene Flüssigkeitsvolumen betrug für jedes Gefäß $3 \mathrm{~m} /$. Es setzte sich zusammen aus:

$0,5 \mathrm{~m} /$ Lungenhomogenat, $2 \mathrm{~m} /$ Ringer-Bikarbonatlösung $(0,15-\mathrm{m}$ $\mathrm{NaHCO}_{3}$ ) sowie die entsprechende Menge Tween 20. Mit abnehmender Tween-Konzentration wurde das Flüssigkeitsvolumen im Gefäß mit dest. Wasser auf $3 \mathrm{ml}$ ergänzt. In die Kontrollgefäße wurden statt Lungenhomogenat die entsprechenden Mengen dest. Wasser hinzugefügt.

Vor Beginn des Versuches wurden die Gefäße 5 Minuten lang mit einem aus 5\% $\mathrm{CO}_{2}$ und $95 \% \mathrm{~N}_{2}$ bestehenden Gasgemisch durchströmt und nach Verschluß der Gefäße durch Schütteln in einem Wasserbad, das genau auf $37^{\circ}$ eingestellt war, der Temperaturausgleich abgewartet.

Innerhalb 60 Minuten wurden alle 10 Minuten die Druckdifferenzen abgelesen. Aus den Druckdifferenzen ergaben sich durch Umrechnung die durch Spaltung

Tab. 1

Spaltung von Tween 20 durch $0,25 \mathrm{~g}$ Homogenat von Kaninchenlunge. Werte in $\mu \mathrm{ml} \mathrm{CO}$. (Kontrollwerte in Klammein)

\begin{tabular}{|c|c|c|c|c|c|c|}
\hline \multirow{2}{*}{$\begin{array}{c}\text { mMol } \\
\text { Tween } 20 \\
\text { pro Arisatz }\end{array}$} & \multicolumn{3}{|c|}{ Minuten nach } & \multicolumn{3}{|c|}{ Versuchs'eginn } \\
\hline & 10 & 20 & 30 & 40 & 50 & 60 \\
\hline 0,407 & $\begin{array}{l}78,21 \\
(2,34)\end{array}$ & $\begin{array}{r}122,43 \\
(3,92)\end{array}$ & $\begin{array}{r}157,41 \\
(5,50)\end{array}$ & $\begin{array}{r}186,03 \\
(5,53)\end{array}$ & $\begin{array}{r}209,87 \\
(6,29)\end{array}$ & $\begin{array}{r}232,13 \\
(4,74)\end{array}$ \\
\hline 0,244 & $\begin{array}{l}73,04 \\
(4,53)\end{array}$ & $\begin{array}{c}122,84 \\
(5,28)\end{array}$ & $\begin{array}{c}159,41 \\
(5,28)\end{array}$ & $\begin{array}{c}185,84 \\
(5,28)\end{array}$ & $\begin{array}{r}207,61 \\
(5,28)\end{array}$ & $\begin{array}{r}224,72 \\
(3,77)\end{array}$ \\
\hline 0,081 & $\begin{array}{l}61,95 \\
(2,36)\end{array}$ & $\begin{array}{l}95,60 \\
(6,36)\end{array}$ & $\begin{array}{c}120,84 \\
(6,33)\end{array}$ & $\begin{array}{r}138,43 \\
(7,13)\end{array}$ & $\begin{array}{r}150,67 \\
(7,87)\end{array}$ & $\begin{array}{r}162,14 \\
(9,44)\end{array}$ \\
\hline 0,065 & $\begin{array}{l}53,58 \\
(4,51)\end{array}$ & $\begin{array}{l}83,08 \\
(6,02)\end{array}$ & $\begin{array}{l}105,60 \\
(10,57)\end{array}$ & $\begin{array}{c}122,71 \\
(7,49)\end{array}$ & $\begin{array}{r}137,46 \\
(8,98)\end{array}$ & $\begin{array}{r}147,59 \\
(6,75)\end{array}$ \\
\hline 0,048 & $\begin{array}{l}44,09 \\
(2,40)\end{array}$ & $\begin{array}{l}67,71 \\
(2,40)\end{array}$ & $\begin{array}{l}85,83 \\
(1,61)\end{array}$ & $\begin{array}{r}101,58 \\
(1,61)\end{array}$ & $\begin{array}{r}113,40 \\
(3,22)\end{array}$ & $\begin{array}{r}122,06 \\
(1,61)\end{array}$ \\
\hline 0,032 & $\begin{array}{l}36,99 \\
(1,52)\end{array}$ & $\begin{array}{l}56,66 \\
(3,82)\end{array}$ & $\begin{array}{l}71,64 \\
(6,12)\end{array}$ & $\begin{array}{l}87,39 \\
(4,58)\end{array}$ & $\begin{array}{r}102,34 \\
(6,10)\end{array}$ & $\begin{array}{r}110,98 \\
(4,58)\end{array}$ \\
\hline 0,016 & $\begin{array}{l}24,90 \\
(0)\end{array}$ & $\begin{array}{c}41,98 \\
(3,00)\end{array}$ & $\begin{array}{l}53,63 \\
(6,00)\end{array}$ & $\begin{array}{l}62,20 \\
(4,50)\end{array}$ & $\begin{array}{l}70,76 \\
(4,50)\end{array}$ & $\begin{array}{l}77,01 \\
(4,50)\end{array}$ \\
\hline 0,008 & $\begin{array}{l}15,51 \\
(3,81)\end{array}$ & $\begin{array}{l}24,05 \\
(5,23)\end{array}$ & $\begin{array}{l}31,86 \\
(5,27)\end{array}$ & $\begin{array}{l}34,17 \\
(3,77)\end{array}$ & $\begin{array}{l}36,49 \\
(4,54)\end{array}$ & $\begin{array}{c}38,04 \\
(4,54)\end{array}$ \\
\hline 0,004 & $\begin{array}{l}3,06 \\
(0)\end{array}$ & $\begin{array}{l}9,30 \\
(0)\end{array}$ & $\begin{array}{l}13,20 \\
(1,49)\end{array}$ & $\begin{array}{l}17,10 \\
(1,49)\end{array}$ & $\begin{array}{l}20,21 \\
(1,49)\end{array}$ & $\begin{array}{l}23,32 \\
(1,49)\end{array}$ \\
\hline 0,0008 & $\begin{array}{l}1,60 \\
(0)\end{array}$ & $\begin{array}{c}3,98 \\
(3,10)\end{array}$ & $\begin{array}{c}5,56 \\
(4,71)\end{array}$ & $\begin{array}{c}8,74 \\
(4,70)\end{array}$ & $\begin{array}{l}10,33 \\
(5,55)\end{array}$ & $\begin{array}{l}11,92 \\
(6,36)\end{array}$ \\
\hline
\end{tabular}

von Tween 20 mittels Lipase frei werdenden $\mu l \mathrm{CO}_{2}$. Die in der Tabelle aufgeführten Zahlen stellen die Mittelwerte aus mindestens zwei Einzelbeobachtungen dar. Die nach Abzug der $\mathrm{CO}_{2}$-Werte der Kontrollversuche daraus resultierenden Werte für $\mathrm{CO}_{2}$ wurden graphisch ermittelt.

\section{Ergebnisse}

Die folgende Abbildung 1 und Tabelle 1 zeigen die Resultate bei unterschiedlichen Versuchsanordnungen.

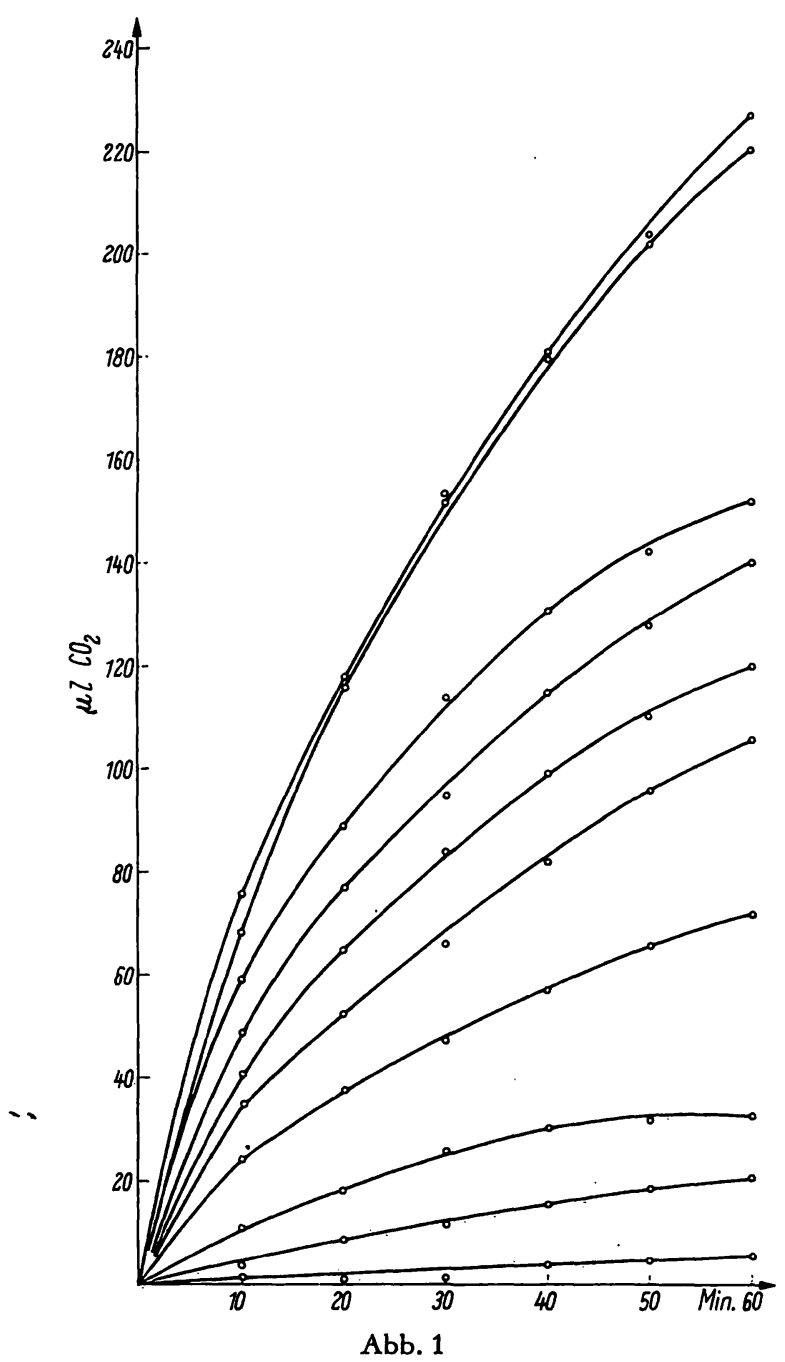

Zeitlicher Ablauf der fermentativen Spaltung von 0,407-0,0008 $\mathrm{mMol}$ „Tween $20^{\text {“ }}$ durch $0,25 \mathrm{~g}$ Lungenhomogenat bei $37^{\circ}$

\section{Literatur}

1. Arlas-Goldschmidt GmbH, Essen, Lieferprogramm (1962). 2. Berenblum, J., Cancer. Res. 1: 44, 807 (1941). - 3. Setälä, K., H. Setälä, L. Merenmies und P. Holstr, Zschr. Krebsforsch. 61, 534 (1957). - 4. Setälä, K., K. Dammert, H. Setälä, L. MerenMIES und P. Holstr, Zschr. Krebsforsch. 61, 548 (1957). - 5. Setälä, K., P. Holsti, S. Lundbom, L. Merenmies und K. Dammert, Zschr. Krebsforsch. 61, 569 (1957). - 6. Berenblum, J. und N. Haran, Cancer Res. 15, 510 (1955). - 7. Wong, T. W., D. S. Juras und R. U. Wissler, J. Nat. Cancer Inst. 22, 363 (1959). - 8. Shubik, P., G. Della Porta und K. Spencer, Acta cancr. Budapest 15, 232 (1959). - 9. HUEPER, W. C. und W. W. Payne, Arch. environment Health 6, 484 (1963). - 10. Sieber, W. Hoppe-Seyler's Z. physiol. Chem. 55, 177 (1908). - 11. BRAdLEY, C. J., J. biol. Chemistry 13, 407 (1913). - 12. Hamsik, K., HoppeSeyler's Z. physiol. Chem. 90, 489 (1914). - 13. Ammon, R. und W. Dirschel, Fermente, Hormone, Vitamine, Georg Thieme Verlag, Stuttgart (1948). - 14. WARBuRG, O., Weiterentwicklung der zellphysiologischen Methoden, Georg Thieme Verlag, Stuttgart (1962). 